\section{Acute urinary retention in early pregnancy: how far should the investigation go?}

\author{
Chukwudi O. Okorie \\ Pan African Academy of Christian \\ Surgeons at Tenwek Hospital, \\ Department of Surgery, Bomet, Kenya
}

\begin{abstract}
Acute urinary retention in early pregnancy is a rare but serious problem. In most cases a causative factor can be identified. In the absence of a readily identifiable causative factor following basic investigation, the further diagnostic and treatment approach can be a challenge, as there are no defined guidelines. A case of acute urinary retention in early pregnancy with no readily identifiable cause managed by patient self-intermittent catheterization on outpatient basis is hereby reported.
\end{abstract}

\section{Introduction}

Acute urinary retention in women remains an uncommon event. In the majority of the cases a anatomic causative factor can be identified, however in many other cases the causative factor can be elusive and commonly classified as psychogenic. ${ }^{1,2}$ Acute urinary retention in early pregnancy is even rarer with reported cases commonly attributed to obstructing bladder or pelvic masses,${ }^{3,4,5}$ vulval edema ${ }^{6}$ cervical pregnancy $^{7}$ or to the retroverted uterus. ${ }^{4,8-10}$ In the rare occasion of acute urinary retention in early pregnancy where no obvious pathology is readily identifiable, the extent of diagnostic investigation and treatment approach remains undefined and can be a challenge to the treating physician. Here is reported the use of intermittent self catheterization on outpatient basis for the treatment of acute urinary retention in early pregnancy after basic investigation ruled out any serious or life threatening pathology in the absence of a definitive causative factor.

\section{Case Report}

A 20-year-old gravida 1 para 0 woman of 13 weeks gestation presented to the urology clinic on an indwelling Foley catheter of $23 \mathrm{~h}$ duration. The first episode of acute urinary retention during which the patient was completely unable to urinate was three days prior to presentation for which patient was drained of $1100 \mathrm{cc}$ of urine at a district clinic using a straight catheter. Urinalysis and pelvic exam done at this clinic of initial presentation didn't reveal any infection or any obvious pelvic abnormality. The morning of the following day, there was a repeat inability to urinate and at the same peripheral clinic an indwelling catheter was now inserted with 1300 cc of urine drained into the urinary bag and patient started on antibiotics. Investigation on presentation included medical, surgical, medication and social history that didn't reveal any problem in this psychologically stable patient; the patient had enjoyed good health prior to this illness with no prior urinary tract symptoms (LUTS) and no history of pelvic surgery or surgery elsewhere on the body. The patient was not on any medication, not constipated and neurological examination was of no abnormal findings. The uterus was anteverted on both pelvic and ultrasound examination and there were no fibroids, prolapse, adnexal or pelvic mass. Vaginal examination with and without Valsalva maneuver failed to show abnormality of cervical position or that of the anterior vaginal wall. Complete blood count, urinalysis and human immunodeficiency virus (HIV) test were all of no abnormal findings. Obstetric and urinary system ultrasound showed a singleton cephalic fetus without any other abnormal findings in the above systems. Urethro-cystoscopy didn't reveal any obstructing lesion. The catheter was then not reinserted and the patient was taught how to do selfcatheterization should the urinary retention recur with plan to not admit patient to the ward but rather to closely monitor her on outpatient basis. She was given several sterile size 14Fr straight catheters for single use on any occasion of further urinary retention. On follow up, the patient had recurrent urinary retention on day 5 and 9 from time of urology review after which there was no more incidence of urinary retention until normal vaginal delivery. Urine culture done two weeks from presentation while the patient was already on self-catheterization as needed didn't grow any microorganism. On follow-up after delivery, the patient has been voiding well. Control urinalysis done at one month of follow-up was without abnormal findings. Control ultrasound of the kidneys and bladder was done at one and three months of follow-up with normal findings and no residual urine. Uroflowmetry or any other further investigations were not done. The patient has been followed up for 18 months now.

\section{Discussion}

Acute urinary retention in women and especially in pregnancy is an emergency that should necessitate appropriate work-up towards a definitive diagnosis with subse-
Correspondence: Chukwudi 0. Okorie, Department of Surgery, Federal Teaching Hospital P.M.B 102 Abakaliki, Ebonyi State, Nigeria.

E-mail: okorieco@mail.ru; okorie@paacs.net

Key words: acute urinary retention, urinary retention in pregnancy, idiopathic urinary retention, female urinary retention.

Conflict of interests: the author declares no potential conflict of interests.

Received for publication: 1 October 2011.

Revision received: 1 March 2012.

Accepted for publication: 11 May 2012.

This work is licensed under a Creative Commons Attribution NonCommercial 3.0 License (CC BYNC 3.0).

(C) Copyright C.O. Okorie et al., 2012

Licensee PAGEPress, Italy

Urogynaecologia 2012; 26:e5

doi:10.4081/uij.2012.e5

quent treatment as need be. This is particularly important as lack of recognition and proper treatment of a serious underlying cause of acute urinary retention in pregnancy can be a threat to the life of the mother and fetus. Hence all cases of acute urinary retention in pregnancy should be taken very seriously with adequate diagnostic workup and treatment instituted. There is no simple diagnostic test or guideline to identify the cause of acute urinary retention in pregnancy hence it is for each physician to use adequate history, physical examination and ancillary tests as need be to arrive at possible diagnosis but without risk to the mother and fetus. When faced with a situation whereby no readily causative factor is identifiable following basic investigation, the extent of further diagnostic workup and treatment plan can be challenging. In the present case, basic diagnostic workup ruled out presence of any obstructing mass as well as any life threatening pathology. There was no obvious compelling reason to keep the patient in the hospital for observation or to order further investigations hence patient was taught sterile intermittent catheterization and managed on outpatient basis with no complication. This approach was safe, cost effective and convenient to the patient. Hence it can be recommended that in cases of acute urinary retention in pregnancy, basic Work up on presentation should probably include: i) Adequate medical/surgical/medication and social history with particular attention to any presence of symptoms of lower urinary tract and neurological dysfunction; ii) Adequate physical examination of the surrounding organs to the blad- 
der and urethra for any intra vaginal, rectal or pelvic obstructing lesion; iii) Urinalysis and urine culture; iv) Ultrasound of kidney and pelvic organs with measurement of residual urine; v) Urethrocystoscopy to exclude any obstructing lesion that could have been missed on ultrasound.

Subsequent treatment should certainly depend on the final diagnosis after appropriate investigation, however relief of urinary retention should be promptly instituted either through continuous or intermittent catheterization as in this case.

High on the list of differential diagnoses for this case was the possible presence of a neurological problem. Neurological problems like multiple sclerosis, Fowler's syndrome are among possible considerations for unexplained acute urinary retention in young women. ${ }^{11}$ The patient didn't have any noted neurological deficits and after the short episode of recurrent urinary retention that was relieved and managed with self-intermittent catheterization, didn't have any repeated urinary problem. Urodynamic studies could certainly play a significant role in the further diagnosis of young women with acute urinary retention. However urodynamic studies done during acute urinary retention in pregnancy have not been clinically useful. ${ }^{12}$ Since LUTS that developed during pregnancy mostly return to normal soon after delivery, ${ }^{12}$ it may then be reasonable to include urodynamic studies in the further investigation of those with persisting LUTS or that had such pre-pregnancy.

Retroversion of the uterus, which is the more frequently diagnosed cause of acute urinary retention in pregnancy in the absence of obstructing lesions, can be transient. It could be theoretically possible that episodes of urinary retention can occur during such transient periods. Above notwithstanding, when there is no obvious indication at time of presentation to hospitalize a patient for more extensive investigation or for observation as in this case report, outpatient management with intermittent catheterization still seems a rational approach. It is important however to emphasize to the patient of the need to immediately present back to the hospital for any prolonged retention or occurrence of new symptoms that could be potentially a threat to the wellbeing of either the patient or the fetus. For close follow-up, mobile phone was additionally used to maintain contact with the patient as above has been proven to be effective in patient monitoring. ${ }^{13}$

\section{Conclusions}

In any case of acute urinary retention in pregnancy, basic investigation on presentation should include: i) adequate medical/surgical/ medication and social history; ii) physical examination of nervous, genito-urinary and lower intestinal systems; iii) urinalysis and urine culture; iv) ultrasound of pelvic organs and urethro-cystoscopy. If there is no readily identifiable cause following this basic investigation in a situation of a stable patient, treatment could be started on outpatient basis with intermittent catheterization. Close follow-up should however be ensured to prevent any unforeseen circumstances.

\section{References}

1. Doran J, Roberts M. Acute urinary retention in the female. Br J Urol 1976;47:79396.

2. Swinn MJ, Wiseman 0J, Lowe E, et al. The cause and natural history of isolated urinary retention in young women. $\mathrm{J}$ Urol 2002;167:151-6.

3. Goldberg KA, Kwart AM. Intermittent urinary retention in first trimester of pregnancy. Urology 1981;17:270-1.

4. Hansen JH, Asmussen M. Acute urinary retention in first trimester of pregnancy. Acta Obstet Gynecol Scand 1985;64:279-80.

5. Chauleur C, Vulliez L, Seffert P. Acute urine retention in early pregnancy resulting from fibroid incarceration: proposition for management. Fertil Steril 2008;90:1198.e7-10.

6. Yellamareddygari S, Ahluwalia A. Acute vulval oedema with urinary retention in pregnancy. J Obstet Gynaecol 2006;26:816.

7. Heazell AEP, Dwarakanath LS, Sundar K. An unusual cause of urinary retention in early pregnancy. Am J Obstet Gynecol 2004;191: 364-5.

8. Vikram P, Ritesh V, Nerli RB, et al. Acute urinary retention in pregnancy. Recent Res Sci Technol 2010;2:53-4.

9. Suzuki S, Ono S, Satomi M. Recurrence of urinary retention secondary to retroverted gravid uterus. North Am J Med Sci 2009;1: 54-7.

10. Silva PD, Berberich W. Retroverted impacted gravid uterus with acute urinary retention: report of two cases and a review of literature. Obstet Gynecol 1986;68:121-3.

11. Fowler CJ. Neurological disorders of micturition and their treatment. Brain 1999; 122:1213-31.

12. FitzGerald MP, Graziano S. Anatomic and functional changes of the lower urinary tract during pregnancy. Urol Clin North Am 2007;34:7-12.

13. Okorie CO, Pisters LL, Ndasi HT, Fekadu A. A simplified protocol for evaluating and monitoring urethral stricture patients minimizes cost without compromising patient outcome. Trop Doc 2010;40:134-7. 